\title{
Quaternionic equation for electromagnetic fields in inhomogeneous media
}

\author{
Vladislav V. Kravchenko \\ Depto. de Telecomunicaciones, \\ Escuela Superior de Ingeniería Mecánica y Eléctrica, \\ Instituto Politécnico Nacional, \\ C.P.07738, D.F., MEXICO \\ e-mail: vkravche@maya.esimez.ipn.mx
}

October 25, 2001

\begin{abstract}
We show that the Maxwell equations for arbitrary inhomogeneous media are equivalent to a single quaternionic equation which can be considered as a generalization of the Vekua equation for generalized analytic functions.
\end{abstract}

\section{Introduction}

Quaternionic reformulation of the Maxwell equations in a vacuum is quite well known (see, for example, [1], [6], [7], [13]). The system of Maxwell equations

$$
\begin{aligned}
& \operatorname{div} \mathbf{D}=\operatorname{div} \mathbf{B}=0 \\
& \operatorname{rot} \mathbf{E}=-\partial_{t} \mathbf{B}, \quad \operatorname{rot} \mathbf{H}=\partial_{t} \mathbf{D},
\end{aligned}
$$

where $D=\varepsilon_{0} E$ and $B=\mu_{0} H$ ( $\varepsilon_{0}$ and $\mu_{0}$ are the permittivity and permeability of free space), is equivalent to the quaternionic equation

$$
\left(\frac{1}{c_{0}} \partial_{t}+i D\right) \vec{f}=0
$$

where $c_{0}$ is the speed of light in a vacuum, $D$ is the Moisil-Theodoresco operator (see the definition below) and $\vec{f}$ is a purely vectorial complex quaternion. The equivalence between (11) and (2) can be established by putting

$$
\operatorname{Re} \vec{f}=\sqrt{\varepsilon_{0}} E \quad \text { and } \quad \operatorname{Im} \vec{f}=\sqrt{\mu_{0}} H .
$$

The quaternionic approach to Maxwell's equations for homogeneous media was intensively used in a number of works (e.g., [8], [9], [10], [13]), but even the 
question as to how to write the Maxwell equations for arbitrary inhomogeneous media in a compact quaternionic form remained open until recently (an attempt in this direction can be found in [5, Section 4.5]). In [12] such a reformulation was proposed in the case of a time-harmonic electromagnetic field and in [11] for the time-dependent case. Here we make one additional step which leads us to Maxwell's system in the form of a single quaternionic equation, which can be considered as a generalization of the well known in complex analysis Vekua equation describing generalized analytic functions [15].

\section{Preliminaries}

We will consider continuously differentiable functions of four variables $\left(t, x_{1}, x_{2}, x_{3}\right)$ with values in the algebra of complex quaternions $\mathbb{H}(\mathbb{C})$. By $D$ we denote the operator $D=\sum_{k=1}^{3} i_{k} \partial_{k}$. Here $\partial_{k}=\frac{\partial}{\partial x_{k}}$ and $i_{k}$ are the imaginary quaternionic units. Let us notice the following property of the operator $D$. Let $\varphi$ be a scalar complex function and $g$ be an $\mathbb{H}(\mathbb{C})$-valued function. Then

$$
D[\varphi \cdot g]=D[\varphi] \cdot g+\varphi \cdot D[g] .
$$

Taking into account that $D[\varphi]=\operatorname{grad} \varphi=i_{1} \partial_{1} \varphi+i_{2} \partial_{2} \varphi+i_{3} \partial_{3} \varphi$ and assuming that $\varphi$ is different from zero we can rewrite (3) in the form

$$
\left(D+\frac{\operatorname{grad} \varphi}{\varphi}\right) g=\frac{1}{\varphi} D[\varphi \cdot g] .
$$

We will use the following notations for the operators of multiplication from the left-hand side and from the right-hand side

$$
{ }^{\alpha} M g:=\alpha \cdot g \quad \text { and } \quad M^{\alpha} g:=g \cdot \alpha,
$$

where $\alpha \in \mathbb{H}(\mathbb{C})$. The usual complex conjugation we denote by "**. Vectors from $\mathbb{C}^{3}$ are identified with purely vectorial complex quaternions. Note that for an $\mathbb{H}(\mathbb{C})$-valued function $g=g_{0}+\vec{g}$ the action of the operator $D$ can be represented as follows

$$
D g=-\operatorname{div} \vec{g}+\operatorname{grad} g_{0}+\operatorname{rot} \vec{g} .
$$

\section{Maxwell equations}

We assume that the relative permittivity $\varepsilon_{r}$ and the relative permeability $\mu_{r}$ of the material are differentiable functions of coordinates $\varepsilon_{r}=\varepsilon_{r}\left(x_{1}, x_{2}, x_{3}\right)$ and $\mu_{r}=\mu_{r}\left(x_{1}, x_{2}, x_{3}\right)$. The permittivity and the permeability of the medium are introduced as follows

$$
\varepsilon=\varepsilon_{0} \varepsilon_{r} \quad \text { and } \quad \mu=\mu_{0} \mu_{r} .
$$


Then Maxwell's equations for an inhomogeneous medium have the form

$$
\begin{gathered}
\operatorname{rot} \mathbf{H}=\varepsilon \partial_{t} \mathbf{E}+\mathbf{j}, \\
\operatorname{rot} \mathbf{E}=-\mu \partial_{t} \mathbf{H}, \\
\operatorname{div}(\varepsilon \mathbf{E})=\rho, \\
\operatorname{div}(\mu \mathbf{H})=0,
\end{gathered}
$$

where all the magnitudes are real. Equations (7) and (8) can be written as follows

$$
\operatorname{div} \mathbf{E}+<\frac{\operatorname{grad} \varepsilon}{\varepsilon}, \mathbf{E}>=\frac{\rho}{\varepsilon}
$$

and

$$
\operatorname{div} \mathbf{H}+<\frac{\operatorname{grad} \mu}{\mu}, \mathbf{H}>=0
$$

where $\langle\cdot, \cdot\rangle$ denotes the usual scalar product. Combining these equations with (5) and (6) we obtain the Maxwell system in the form

$$
D \mathbf{E}=<\frac{\operatorname{grad} \varepsilon}{\varepsilon}, \mathbf{E}>-\mu \partial_{t} \mathbf{H}-\frac{\rho}{\varepsilon}
$$

and

$$
D \mathbf{H}=<\frac{\operatorname{grad} \mu}{\mu}, \mathbf{H}>+\varepsilon \partial_{t} \mathbf{E}+\mathbf{j} .
$$

Let us make a simple observation: the scalar product of two vectors $\vec{p}$ and $\vec{q}$ can be represented as follows

$$
<\vec{p}, \vec{q}>=-\frac{1}{2}\left(\vec{p} M+M^{\vec{p}}\right) \vec{q} .
$$

Using this fact, from (9) and (10) we obtain the pair of equations

$$
\left(D+\frac{1}{2} \frac{\operatorname{grad} \varepsilon}{\varepsilon}\right) \mathbf{E}=-\frac{1}{2} M^{\frac{\operatorname{grad} \varepsilon}{\varepsilon}} \mathbf{E}-\mu \partial_{t} \mathbf{H}-\frac{\rho}{\varepsilon}
$$

and

$$
\left(D+\frac{1}{2} \frac{\operatorname{grad} \mu}{\mu}\right) \mathbf{H}=-\frac{1}{2} M^{\frac{\operatorname{grad} \mu}{\mu}} \mathbf{H}+\varepsilon \partial_{t} \mathbf{E}+\mathbf{j} .
$$

Note that

$$
\frac{1}{2} \frac{\operatorname{grad} \varepsilon}{\varepsilon}=\frac{\operatorname{grad} \sqrt{\varepsilon}}{\sqrt{\varepsilon}}
$$


Then using (伍), equation (11) can be rewritten in the following form

$$
\frac{1}{\sqrt{\varepsilon}} D(\sqrt{\varepsilon} \cdot \mathbf{E})+\mathbf{E} \cdot \vec{\varepsilon}=-\mu \partial_{t} \mathbf{H}-\frac{\rho}{\varepsilon},
$$

where

$$
\vec{\varepsilon}:=\frac{\operatorname{grad} \sqrt{\varepsilon}}{\sqrt{\varepsilon}}
$$

Analogously, (12) takes the form

$$
\frac{1}{\sqrt{\mu}} D(\sqrt{\mu} \cdot \mathbf{H})+\mathbf{H} \cdot \vec{\mu}=\varepsilon \partial_{t} \mathbf{E}+\mathbf{j}
$$

where

$$
\vec{\mu}:=\frac{\operatorname{grad} \sqrt{\mu}}{\sqrt{\mu}}
$$

Introducing the notations

$$
\overrightarrow{\mathcal{E}}:=\sqrt{\varepsilon} \mathbf{E}, \quad \overrightarrow{\mathcal{H}}:=\sqrt{\mu} \mathbf{H}
$$

and multiplying (13) by $\sqrt{\varepsilon}$ and (14) by $\sqrt{\mu}$ we arrive at the equations

$$
\left(D+M^{\vec{\varepsilon}}\right) \overrightarrow{\mathcal{E}}=-\frac{1}{c} \partial_{t} \overrightarrow{\mathcal{H}}-\frac{\rho}{\sqrt{\varepsilon}},
$$

and

$$
\left(D+M^{\vec{\mu}}\right) \overrightarrow{\mathcal{H}}=\frac{1}{c} \partial_{t} \overrightarrow{\mathcal{E}}+\sqrt{\mu} \mathbf{j}
$$

where $c=1 / \sqrt{\varepsilon \mu}$ is the speed of propagation of electromagnetic waves in the medium.

Equations (15) and (16) can be rewritten even in a more elegant form. Consider the function

$$
\vec{f}:=\overrightarrow{\mathcal{E}}+i \overrightarrow{\mathcal{H}}
$$

Let us apply to it the quaternionic Maxwell operator

$$
\frac{1}{c} \partial_{t}+i D
$$

We obtain

$$
\left(\frac{1}{c} \partial_{t}+i D\right) \vec{f}=\frac{1}{c} \partial_{t} \overrightarrow{\mathcal{E}}-D \overrightarrow{\mathcal{H}}+i\left(\frac{1}{c} \partial_{t} \overrightarrow{\mathcal{H}}+D \overrightarrow{\mathcal{E}}\right) .
$$

For the real part of this expression we use equation (16) and for the imaginary part equation (15). Then we have 


$$
\left(\frac{1}{c} \partial_{t}+i D\right) \vec{f}=-i\left(M^{\vec{\varepsilon}} \overrightarrow{\mathcal{E}}+i M^{\vec{\mu}} \overrightarrow{\mathcal{H}}\right)-\sqrt{\mu} \mathbf{j}-\frac{i \rho}{\sqrt{\varepsilon}}
$$

Note that

$$
\overrightarrow{\mathcal{E}}=\frac{1}{2}\left(\vec{f}+\vec{f}^{*}\right) \quad \text { and } \quad \overrightarrow{\mathcal{H}}=\frac{1}{2 i}\left(\vec{f}-\vec{f}^{*}\right)
$$

Hence

$$
M^{\vec{\varepsilon}} \overrightarrow{\mathcal{E}}+i M^{\vec{\mu}} \overrightarrow{\mathcal{H}}=\frac{1}{2}\left(M^{(\vec{\varepsilon}+\vec{\mu})} \vec{f}+M^{(\vec{\varepsilon}-\vec{\mu})} \vec{f}^{*}\right) .
$$

Let us notice that

$$
\vec{\varepsilon}+\vec{\mu}=-\frac{\operatorname{grad} c}{c} \quad \text { and } \quad \vec{\varepsilon}-\vec{\mu}=-\frac{\operatorname{grad} W}{W},
$$

where $W=\sqrt{\mu / \varepsilon}$ is the intrinsic wave impedance of the medium. Denote

$$
\vec{c}:=\frac{\operatorname{grad} \sqrt{c}}{\sqrt{c}} \quad \text { and } \quad \vec{W}:=\frac{\operatorname{grad} \sqrt{W}}{\sqrt{W}} .
$$

Then

$$
M^{\vec{\varepsilon}} \overrightarrow{\mathcal{E}}+i M^{\vec{\mu}} \overrightarrow{\mathcal{H}}=-\left(M^{\vec{c}} \vec{f}+M^{\vec{W}} \vec{f}^{*}\right) .
$$

From (17) we obtain the Maxwell equations for an inhomogeneous medium in the following form

$$
\left(\frac{1}{c} \partial_{t}+i D\right) \vec{f}-M^{i \vec{c}} \vec{f}-M^{i \vec{W}} \vec{f}^{*}=-\left(\sqrt{\mu} \mathbf{j}+\frac{i \rho}{\sqrt{\varepsilon}}\right)
$$

(compare with (2)). This equation is completely equivalent to the Maxwell system (5)-(8) and represents Maxwell's equation for inhomogeneous media in a quaternionic form.

Remark 1 Equation (18) can be considered as a generalization of the well known in complex analysis Vekua equation describing generalized analytic functions [15]. Recently in [14] using the L. Bers approach [9], 3] another quaternionic generalization of the Vekua equation was considered. Probably some of the interesting results discussed in [14] can be obtained for [18) also. Then their physical meaning would be of a great interest.

Acknowledgement 2 This work was supported by CONACYT Project 32424E, Mexico. 


\section{References}

[1] A. V. Berezin, Yu. A. Kurochkin and E. A. Tolkachev Quaternions in relativistic physics. (Nauka y Tekhnika, Minsk, 1989) (in Russian).

[2] L. Bers Theory of pseudo-analytic functions. (New York University, Institute of Mathematics and Mechanics, N.Y., 1953).

[3] L. Bers An outline of the theory of pseudo-analytic functions. Bull. Amer. Math. Soc., v. 62, 291-331 (1956).

[4] K. Gürlebeck and W. Sprößig Quaternionic analysis and elliptic boundary value problems (Akademie-Verlag, Berlin, 1989).

[5] K. Gürlebeck, W. Sprössig Quaternionic and Clifford Calculus for Physicists and Engineers. (John Wiley \& Sons, 1997).

[6] F. Gürsey, H. C. Tze On the role of division, Jordan and related algebras in particle physics. (World Scientific, Singapore, 1996).

[7] K. Imaeda $A$ new formulation of classical electrodynamics. Nuovo Cimento, v. 32 B, \# 1, p. 138-162 (1976).

[8] K.V. Khmelnytskaya, V. V. Kravchenko and H. Oviedo Quaternionic integral representations for electromagnetic fields in chiral media. Telecommunications and Radio Engineering, v. 56, \# 4-5, (2001) (to appear).

[9] K. V. Khmelnytskaya, V. V. Kravchenko, V. S. Rabinovich Métodos del análisis cuaterniónico en los problemas de propagación de ondas electromagnéticas producidas por las fuentes en movimiento. Proceedings of the Second International Congress of Electromechanical and System Engineering Internacional de Ingeniería Electromecánica y de Sistemas, México D.F. (1999).

[10] V. V. Kravchenko On the relation between holomorphic biquaternionic functions and time-harmonic electromagnetic fields. (Deposited in UkrINTEI, 29.12.1992, \#2073 - Uk - 92) (in Russian).

[11] V. V. Kravchenko Applied quaternionic analysis. Maxwell's system and Dirac's equation. World Scientific, "Functional-analytic and complex methods, their interactions, and applications to partial differential equations", Ed. by W. Tutschke, 143-160 (2001).

[12] V. V. Kravchenko Quaternionic reformulation of Maxwell's equations for inhomogeneous media and new solutions. Zeitschrift für Analysis und ihre Anwendungen (to appear).

[13] V. V. Kravchenko and M. V. Shapiro Integral representations for spatial models of mathematical physics (Addison-Wesley Longman Ltd., Pitman Res. Notes in Math. Series, v. 351, London, 1996). 
[14] H. Malonek Generalizing the $(F, G)$-derivative in the sense of Bers. Clifford Algebras and Their Application in Mathematical Physics (V. Dietrich et al. eds.), Kluwer Acad. Publ., 247-257 (1998).

[15] I. N. Vekua Generalized analytic functions. (Pergamon Press, Oxford, 1962). 